\title{
Effective Translation of LTL to Deterministic Rabin Automata: Beyond the (F,G)-Fragment ${ }^{\star}$
}

\author{
Tomáš Babiak, František Blahoudek, Mojmír Křetínský, and Jan Strejček \\ Faculty of Informatics, Masaryk University, Brno, Czech Republic \\ \{xbabiak, xblahoud, kretinsky, strejcek\}@i.muni.cz
}

\begin{abstract}
Some applications of linear temporal logic (LTL) require to translate formulae of the logic to deterministic $\omega$-automata. There are currently two translators producing deterministic automata: 1tl2dstar working for the whole LTL and Rabinizer applicable to LTL(F, G) which is the LTL fragment using only modalities F and G. We present a new translation to deterministic Rabin automata via alternating automata and deterministic transition-based generalized Rabin automata. Our translation applies to a fragment that is strictly larger than $\operatorname{LTL}(F, G)$. Experimental results show that our algorithm can produce significantly smaller automata compared to Rabinizer and 1t12dstar, especially for more complex LTL formulae.
\end{abstract}

\section{Introduction}

Linear temporal logic (LTL) is a popular formalism for specification of behavioral system properties with major applications in the area of model checking [74]. More precisely, LTL is typically used as a human-oriented front-end formalism as LTL formulae are succinct and easy to write and understand. Model checking algorithms usually work with an $\omega$-automaton representing all behaviors violating a given specification formula rather than with the LTL formula directly. Hence, specifications written in the form of LTL formulae are negated and translated to equivalent $\omega$-automata 31. There has been a lot of attention devoted to translation of LTL to nondeterministic Büchi automata (NBA), see for example 9102914] and the research in this direction still continues [1132]. However, there are algorithms that need specifications given by deterministic $\omega$-automata, for example, those for LTL model checking of probabilistic systems 30184] and those for synthesis of reactive modules for LTL specifications [6 26, for a recent survey see 20. As deterministic Büchi automata (DBA) cannot express all the properties expressible in LTL, one has to choose deterministic automata with different acceptance condition.

There are basically two approaches to translation of LTL to deterministic $\omega$-automata. The first one translates LTL to NBA and then it employs Safra's construction [27] (or some of its variants or alternatives like [23 28]) to transform the NBA into a deterministic automaton. This approach is represented by the

\footnotetext{
* This is a full version of the paper accepted to ATVA 2013.
} 
tool lt12dstar [16] which uses an improved Safra's construction [17/18] usually in connection with LTL to NBA translator LTL2BA [14. The main advantage of this approach is its universality: as LTL2BA can translate any LTL formula into an NBA and the Safra's construction can transform any NBA to a deterministic Rabin automaton (DRA), ltl2dstar works for the whole LTL. The main disadvantage is also connected with the universality: the determinization step does not employ the fact that the NBA represents only an LTL definable property. One can easily observe that $1 \mathrm{t} 12 \mathrm{dstar}$ produces unnecessarily large automata, especially for formulae with more fairness subformulae.

The second approach is to avoid Safra's construction. As probabilistic modelcheckers deal with linear arithmetic, they do not profit from symbolically represented deterministic automata of [2422. A few translations of some simple LTL fragments to DBA have been suggested, for example [1]. Recently, a translation of a significantly larger LTL fragment to DRA has been introduced in 19] and subsequently implemented in the tool Rabinizer [13. The algorithm builds a generalized deterministic Rabin automata (GDRA) directly from a formula. A DRA is then produced by a degeneralization procedure. Rabinizer often produces smaller automata than lt12dstar. The main disadvantage is that it works for $\operatorname{LTL}(F, G)$ only, i.e. the LTL fragment containing only temporal operators eventually $(\mathrm{F})$ and always $(\mathrm{G})$. Authors of the translation claim that it can be extended to a fragment containing also the operator next (X).

In this paper, we present another Safraless translation of an LTL fragment to DRA. The translation is influenced by the successful LTL to NBA translation algorithm LTL2BA [14] and it proceeds in the following three steps:

1. A given LTL formula $\varphi$ is translated into a very weak alternating co-Büchi automaton (VWAA) $\mathcal{A}$ as described in [14. If $\varphi$ is an $\operatorname{LTL}\left(\mathrm{F}_{\mathrm{s}}, \mathrm{G}_{\mathrm{s}}\right)$ formula, i.e. any formula which makes use of $F, G$, and their strict variants $F_{s}$ and $\mathrm{G}_{\mathrm{s}}$ as the only temporal operators, then $\mathcal{A}$ satisfies an additional structural condition. We call such automata may/must alternating automata (MMAA).

2. The MMAA $\mathcal{A}$ is translated into a transition-based generalized deterministic Rabin automaton $(T G D R A) \mathcal{G}$. The construction of generalized Rabin pairs of $\mathcal{G}$ is inspired by 19 .

3. Finally, $\mathcal{G}$ is degeneralized into a (state-based) DRA $\mathcal{D}$.

In summary, our contributions are as follows. First, note that the fragment $\operatorname{LTL}\left(F_{s}, G_{s}\right)$ is strictly more expressive than $\operatorname{LTL}(F, G)$. Moreover, it can be shown that our translation works for a fragment even larger than $\operatorname{LTL}\left(F_{s}, G_{s}\right)$ but still smaller than the whole LTL. Second, the translation has a slightly better theoretical bound on the size of produced automata comparing to ltl2dstar, but the same bound as Rabinizer. Experimental results show that, for small formulae, our translation typically produces automata of a smaller or equal size as the other two translators. However, for parametrized formulae, it often produces automata that are significantly smaller. Third, we note that our TGDRA are much smaller than the (state-based) GDRA of Rabinizer [13. We conjecture that algorithms for model checking of probabilistic system, e.g. those in PRISM 21], can be adapted to work with TGDRA as they are adapted to work with GDRA [5]. 


\section{Preliminaries}

This section recalls the notion of linear temporal logic (LTL) [25] and describes the $\omega$-automata used in the following.

Linear Temporal Logic (LTL) The syntax of LTL is defined by

$$
\varphi::=t t|a| \neg \varphi|\varphi \vee \varphi| \varphi \wedge \varphi|\mathrm{X} \varphi| \varphi \mathrm{U} \varphi,
$$

where $t t$ stands for true, a ranges over a countable set AP of atomic propositions, $\mathrm{X}$ and $\mathrm{U}$ are temporal operators called next and until, respectively. An alphabet is a finite set $\Sigma=2^{A P^{\prime}}$, where $A P^{\prime}$ is a finite subset of $A P$. An $\omega$-word (or simply a word) over $\Sigma$ is an infinite sequence of letters $u=u_{0} u_{1} u_{2} \ldots \in \Sigma^{\omega}$. By $u_{i . .}$ we denote the suffix $u_{i . .}=u_{i} u_{i+1} \ldots$

We inductively define when a word $u$ satisfies a formula $\varphi$, written $u \models \varphi$, as follows.

$$
\begin{array}{ll}
u \models t t & \\
u \models a & \text { iff } a \in u_{0} \\
u \models \neg \varphi & \text { iff } u \models \varphi \\
u \models \varphi_{1} \vee \varphi_{2} & \text { iff } u \models \varphi_{1} \text { or } u \models \varphi_{2} \\
u \models \varphi_{1} \wedge \varphi_{2} & \text { iff } u \models \varphi_{1} \text { and } u \models \varphi_{2} \\
u \models \mathrm{X} \varphi & \text { iff } u_{1 . . \models \varphi} \\
u \models \varphi_{1} \cup \varphi_{2} & \text { iff } \exists i \geq 0 .\left(u_{i . .} \models \varphi_{2} \text { and } \forall 0 \leq j<i . u_{j . .} \models \varphi_{1}\right)
\end{array}
$$

Given an alphabet $\Sigma$, a formula $\varphi$ defines the language $L^{\Sigma}(\varphi)=\left\{u \in \Sigma^{\omega} \mid\right.$ $u \models \varphi\}$. We write $L(\varphi)$ instead of $L^{2^{A P(\varphi)}}(\varphi)$, where $A P(\varphi)$ denotes the set of atomic propositions occurring in the formula $\varphi$.

We define derived unary temporal operators eventually $(\mathrm{F})$, always $(\mathrm{G})$, strict eventually $\left(\mathrm{F}_{\mathrm{s}}\right)$, and strict always $\left(\mathrm{G}_{\mathrm{s}}\right)$ by the following equivalences: $\mathrm{F} \varphi \equiv t t \mathrm{U} \varphi$, $\mathrm{G} \varphi \equiv \neg \mathrm{F} \neg \varphi, \mathrm{F}_{\mathrm{s}} \varphi \equiv \mathrm{XF} \varphi$, and $\mathrm{G}_{\mathrm{s}} \varphi \equiv \mathrm{XG} \varphi$.

$\operatorname{LTL}(F, G)$ denotes the LTL fragment consisting of formulae built with temporal operators $F$ and $G$ only. The fragment build with temporal operators $F_{s}$, $\mathrm{G}_{\mathrm{s}}, \mathrm{F}$ and $\mathrm{G}$ is denoted by $\operatorname{LTL}\left(\mathrm{F}_{\mathrm{s}}, \mathrm{G}_{\mathrm{s}}\right)$ as $\mathrm{F} \varphi$ and $\mathrm{G} \varphi$ can be seen as abbreviations for $\varphi \vee F_{s} \varphi$ and $\varphi \wedge G_{s} \varphi$, respectively. Note that $\operatorname{LTL}\left(F_{s}, G_{s}\right)$ is strictly more expressive than $\operatorname{LTL}(\mathrm{F}, \mathrm{G})$ as formulae $\mathrm{F}_{\mathrm{s}} a$ and $\mathrm{G}_{\mathrm{s}} a$ cannot be equivalently expressed in $\operatorname{LTL}(\mathrm{F}, \mathrm{G})$.

An LTL formula is in positive normal form if no operator occurs in the scope of any negation. Each $\operatorname{LTL}\left(F_{s}, G_{s}\right)$ formula can be transformed to this form using De Morgan's laws for $\wedge$ and $\vee$ and the equivalences $\neg \mathrm{F}_{\mathrm{s}} \psi \equiv \mathrm{G}_{\mathrm{s}} \neg \psi, \neg \mathrm{G}_{\mathrm{s}} \psi \equiv \mathrm{F}_{\mathrm{s}} \neg \psi$, $\neg \mathrm{F} \psi \equiv \mathrm{G} \neg \psi$, and $\neg \mathrm{G} \psi \equiv \mathrm{F} \neg \psi$. We say that a formula is temporal if its topmost operator is neither conjunction, nor disjunction (note that $a$ and $\neg a$ are also temporal formulae).

Deterministic Rabin Automata and Their Generalization A semiautomaton is a tuple $\mathcal{T}=\left(S, \Sigma, \delta, s_{I}\right)$, where $S$ is a finite set of states, $\Sigma$ is an 
alphabet, $s_{I} \in S$ is the initial state, and $\delta \subseteq S \times \Sigma \times S$ is a deterministic transition relation, i.e. for each state $s \in S$ and each $\alpha \in \Sigma$, there is at most one state $s^{\prime}$ such that $\left(s, \alpha, s^{\prime}\right) \in \delta$. A triple $\left(s, \alpha, s^{\prime}\right) \in \delta$ is called a transition from $s$ to $s^{\prime}$ labelled by $\alpha$, or an $\alpha$-transition of $s$ leading to $s^{\prime}$. In illustrations, all transitions with the same source state and the same target state are usually depicted by a single edge labelled by a propositional formula $\psi$ over $A P$ representing the corresponding transition labels (e.g. given $\Sigma=2^{\{a, b\}}$, the formula $\psi=a \vee b$ represents labels $\{a\},\{a, b\},\{b\})$.

A run of a semiautomaton $\mathcal{T}$ over a word $u=u_{0} u_{1} \ldots \in \Sigma^{\omega}$ is an infinite sequence $\sigma=\left(s_{0}, u_{0}, s_{1}\right)\left(s_{1}, u_{1}, s_{2}\right) \ldots \in \delta^{\omega}$ of transitions such that $s_{0}=s_{I}$. By $\operatorname{Inf} f_{t}(\sigma)$ (resp. $\left.\operatorname{Inf} f_{s}(\sigma)\right)$ we denote the set of transitions (resp. states) occurring infinitely often in $\sigma$. For each word $u \in \Sigma^{\omega}$, a semiautomaton has at most one run over $u$ denoted by $\sigma(u)$.

A deterministic Rabin automaton (DRA) is a tuple $\mathcal{D}=\left(S, \Sigma, \delta, s_{I}, \mathcal{R}\right)$, where $\left(S, \Sigma, \delta, s_{I}\right)$ is a semiautomaton and $\mathcal{R} \subseteq 2^{S} \times 2^{S}$ is a finite set of Rabin pairs. Runs of $\mathcal{D}$ are runs of the semiautomaton. A run $\sigma$ satisfies a Rabin pair $(K, L) \in \mathcal{R}$ if $\operatorname{Inf}_{s}(\sigma) \cap K=\emptyset$ and $\operatorname{Inf}_{s}(\sigma) \cap L \neq \emptyset$. A run is accepting if it satisfies some Rabin pair of $\mathcal{R}$. The language of $\mathcal{D}$ is the set $L(\mathcal{D})$ of all words $u \in \Sigma^{\omega}$ such that $\sigma(u)$ is accepting.

A transition-based generalized deterministic Rabin automaton (TGDRA) is a tuple $\mathcal{G}=\left(S, \Sigma, \delta, s_{I}, \mathcal{G R}\right)$, where $\left(S, \Sigma, \delta, s_{I}\right)$ is a semiautomaton and $\mathcal{G R} \subseteq$ $2^{\delta} \times 2^{2^{\delta}}$ is a finite set of generalized Rabin pairs. Runs of $\mathcal{G}$ are runs of the semiautomaton. A run $\sigma$ satisfies a generalized Rabin pair $\left(K,\left\{L_{j}\right\}_{j \in J}\right) \in \mathcal{G} \mathcal{R}$ if $\operatorname{Inf}_{t}(\sigma) \cap K=\emptyset$ and, for each $j \in J, \operatorname{Inf}_{t}(\sigma) \cap L_{j} \neq \emptyset$. A run is accepting if it satisfies some generalized Rabin pair of $\mathcal{G R}$. The language of $\mathcal{G}$ is the set $L(\mathcal{G})$ of all words $u \in \Sigma^{\omega}$ such that $\sigma(u)$ is accepting.

A generalization of DRA called generalized deterministic Rabin automata (GDRA) has been considered in 1913. The accepting condition of GDRA is a positive Boolean combination (in disjunctive normal form) of Rabin pairs. A run $\sigma$ is accepting if $\sigma$ satisfies this condition.

Very Weak Alternating Automata and Their Subclass A very weak alternating co-Büchi automaton (VWAA) $\mathcal{A}$ is a tuple $(S, \Sigma, \delta, I, F)$, where $S$ is a finite set of states, subsets $c \subseteq S$ are called configurations, $\Sigma$ is an alphabet, $\delta \subseteq S \times \Sigma \times 2^{S}$ is an alternating transition relation, $I \subseteq 2^{S}$ is a non-empty set of initial configurations, $F \subseteq S$ is a set of co-Büchi accepting states, and there exists a partial order on $S$ such that, for every transition $(s, \alpha, c) \in \delta$, all the states of $c$ are lower or equal to $s$.

A triple $(s, \alpha, c) \in \delta$ is called a transition from $s$ to $c$ labelled by $\alpha$, or an $\alpha$-transition of $s$. We say that $s$ is the source state and $c$ the target configuration of the transition. A transition is looping if the target configuration contains the source state, i.e. $s \in c$. A transition is called a selfloop if its target configuration contains the source state only, i.e. $c=\{s\}$.

Figure 1(a) shows a VWAA that accepts the language described by the formula $\mathrm{G}\left(\mathrm{F}_{\mathrm{s}} a \wedge \mathrm{F}_{\mathrm{s}} b\right) \vee \mathrm{G} b$. Transitions are depicted by branching edges. If a target 


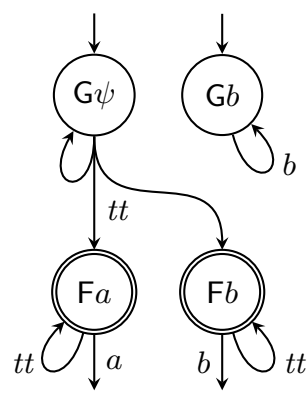

(a)

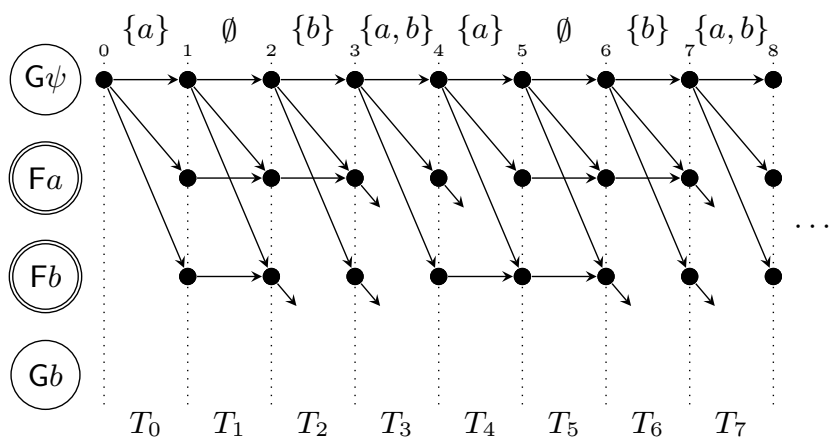

(b)

Fig. 1. (a) A VWAA (and also MMAA) corresponding to formula $\mathrm{G} \psi \vee \mathrm{G} b$, where $\psi=\mathrm{F}_{\mathrm{s}} a \wedge \mathrm{F}_{\mathrm{s}} b$. (b) An accepting run of the automaton over $(\{a\} \emptyset\{b\}\{a, b\})^{\omega}$.

configuration is empty, the corresponding edge leads to an empty space. We often depict all transitions with the same source state and the same target configuration by a single edge (as for semiautomata). Each initial configuration is represented by a possibly branching unlabelled edge leading from an empty space to the states of the configuration. Co-Büchi accepting states are double circled.

A multitransition $T$ with a label $\alpha$ is a set of transitions with the same label and such that the source states of the transitions are pairwise different. A source configuration of $T$, denoted by $\operatorname{dom}(T)$, is the set of source states of transitions in $T$. A target configuration of $T$, denoted by range $(T)$, is the union of target configurations of transitions in $T$. We define a multitransition relation $\Delta \subseteq 2^{S} \times \Sigma \times 2^{S}$ as

$$
\Delta=\{(\operatorname{dom}(T), \alpha, \operatorname{range}(T)) \mid \text { there exists a multitransition } T \text { with label } \alpha\} .
$$

A run $\rho$ of a VWAA $\mathcal{A}$ over a word $w=w_{0} w_{1} \ldots \in \Sigma^{\omega}$ is an infinite sequence $\rho=T_{0} T_{1} \ldots$ of multitransitions of $\mathcal{A}$ such that $\operatorname{dom}\left(T_{0}\right)$ is an initial configuration of $\mathcal{A}$ and, for each $i \geq 0, T_{i}$ is labelled by $w_{i}$ and $\operatorname{range}\left(T_{i}\right)=\operatorname{dom}\left(T_{i+1}\right)$.

A run can be represented as a directed acyclic graph (DAG). For example, the DAG of Figure 1(b) represents a run of the VWAA of Figure 1(a). The dotted lines divide the DAG into segments corresponding to multitransitions. Each transition of a multitransition is represented by edges leading across the corresponding segment from the starting state to states of the target configuration. As our alternating automata are very weak, we can order the states in a way that all edges in any DAG go only to the same or a lower row.

An accepting run corresponds to a DAG where each branch contains only finitely many states from $F$. Formally, the run $\rho$ is accepting if it has no suffix where, for some co-Büchi accepting state $f \in F$, each multitransition contains a looping transition from $f$. The language of $\mathcal{A}$ is the set $L(\mathcal{A})=\left\{w \in \Sigma^{\omega} \mid\right.$ $\mathcal{A}$ has an accepting run of over $w\}$. By $\operatorname{Inf}_{s}(\rho)$ we denote the set of states that occur in $\operatorname{dom}\left(T_{i}\right)$ for infinitely many indices $i$. 
Definition 1. A may/must alternating automaton (MMAA) is a VWAA where each state fits into one of the following three categories:

1. May-states - states with a selfloop for each $\alpha \in \Sigma$. A run that enters such a state may wait in the state for an arbitrary number of steps.

2. Must-states - every transition of a must-state is looping. A run that enters such a state can never leave it. In other words, the run must stay there.

3. Loopless states - states that have no looping transitions and no predecessors. They can appear only in initial configurations (or they are unreachable).

The automaton of Figure $1(\mathrm{a})$ is an MMAA with must-states $\mathrm{G} \psi, \mathrm{G} b$ and may-states $\mathrm{F} a, \mathrm{~F} b$.

We always assume that the set $F$ of an MMAA coincides with the set of all may-states of the automaton. This assumption is justified by the following observations:

- There are no looping transitions of loopless states. Hence, removing all loopless states from $F$ has no effect on acceptance of any run.

- All transitions leading from must-states are looping. Hence, if a run contains a must-state that is in $F$, then the run is non-accepting. Removing all muststates in $F$ together with their adjacent transitions from an MMAA has no effect on its accepting runs.

- Every may-state has selfloops for all $\alpha \in \Sigma$. If such a state is not in $F$, we can always apply these selfloops without violating acceptance of any run. We can also remove these states from all the target configurations of all transitions of an MMAA without affecting its language.

\section{Translation of $\operatorname{LTL}\left(\mathrm{F}_{\mathrm{s}}, \mathrm{G}_{\mathrm{s}}\right)$ to MMAA}

We present the standard translation of LTL to VWAA [14 restricted to the fragment $\operatorname{LTL}\left(\mathrm{F}_{\mathrm{s}}, \mathrm{G}_{\mathrm{s}}\right)$. In this section, we treat the transition relation $\delta \subseteq S \times$ $\Sigma \times 2^{S}$ of a VWAA as a function $\delta: S \times \Sigma \rightarrow 2^{2^{S}}$, where $c \in \delta(s, \alpha)$ means $(s, \alpha, c) \in \delta$. Further, we consider $\mathrm{G} \psi$ and $\mathrm{F} \psi$ to be subformulae of $\mathrm{G}_{\mathrm{s}} \psi$ and $\mathrm{F}_{\mathrm{s}} \psi$, respectively. This is justified by equivalences $\mathrm{G}_{\mathrm{s}} \psi \equiv \mathrm{XG} \psi$ and $\mathrm{F}_{\mathrm{s}} \psi \equiv \mathrm{XF} \psi$. Recall that a formula is called temporal if its topmost operator is neither conjunction, nor disjunction (note that $a$ and $\neg a$ are also temporal formulae).

Let $\varphi$ be an $\operatorname{LTL}\left(\mathrm{F}_{\mathrm{s}}, \mathrm{G}_{\mathrm{s}}\right)$ formula in positive normal form. An equivalent VWAA is constructed as $\mathcal{A}_{\varphi}=(Q, \Sigma, \delta, I, F)$, where

$-Q$ is the set of temporal subformulae of $\varphi$,

$-\Sigma=2^{A P(\varphi)}$,

$-\delta$ is defined as

$$
\begin{aligned}
\delta(t t, \alpha) & =\{\emptyset\} \\
\delta(\neg t t, \alpha) & =\emptyset \\
\delta\left(\mathrm{G}_{\mathrm{s}} \psi, \alpha\right) & =\{\{\mathrm{G} \psi\}\} \\
\delta\left(\mathrm{F}_{\mathrm{s}} \psi, \alpha\right) & =\{\{\mathrm{F} \psi\}\}
\end{aligned}
$$$$
\begin{aligned}
\delta(a, \alpha) & =\{\emptyset\} \text { if } a \in \alpha, \emptyset \text { otherwise } \\
\delta(\neg a, \alpha) & =\{\emptyset\} \text { if } a \notin \alpha, \emptyset \text { otherwise } \\
\delta(\mathrm{G} \psi, \alpha) & =\{c \cup\{\mathrm{G} \psi\} \mid c \in \bar{\delta}(\psi, \alpha)\} \\
\delta(\mathrm{F} \psi, \alpha) & =\{\{\mathrm{F} \psi\}\} \cup \bar{\delta}(\psi, \alpha), \text { where }
\end{aligned}
$$ 


$$
\begin{aligned}
\bar{\delta}(\psi, \alpha) & =\delta(\psi, \alpha) \text { if } \psi \text { is a temporal formula } \\
\bar{\delta}\left(\psi_{1} \vee \psi_{2}, \alpha\right) & =\bar{\delta}\left(\psi_{1}, \alpha\right) \cup \bar{\delta}\left(\psi_{2}, \alpha\right) \\
\bar{\delta}\left(\psi_{1} \wedge \psi_{2}, \alpha\right) & =\left\{c_{1} \cup c_{2} \mid c_{1} \in \bar{\delta}\left(\psi_{1}, \alpha\right) \text { and } c_{2} \in \bar{\delta}\left(\psi_{2}, \alpha\right)\right\},
\end{aligned}
$$

- $I=\bar{\varphi}$ where $\bar{\varphi}$ is defined as

$$
\begin{aligned}
\bar{\psi} & =\{\{\psi\}\} \text { if } \psi \text { is a temporal formula } \\
\frac{\psi_{1} \vee \psi_{2}}{\psi_{1} \wedge \psi_{2}} & =\left\{\mathcal{\psi}_{1} \cup \overline{\psi_{2}} \cup O_{2} \mid O_{1} \in \overline{\psi_{1}} \text { and } O_{2} \in \overline{\psi_{2}}\right\}, \text { and }
\end{aligned}
$$

- $F \subseteq Q$ is the set of all subformulae of the form $\mathrm{F} \psi$ in $Q$.

Using the partial order "is a subformula of" on states, one can easily prove that $\mathcal{A}_{\varphi}$ is a VWAA. Moreover, all the states of the form $\mathrm{G} \psi$ are must-states and all the states of the form $\mathrm{F} \psi$ are may-states. States of other forms are loopless and they are unreachable unless they appear in $I$. Hence, the constructed automaton is also an MMAA. Figure 1(a) shows an MMAA produced by the translation of formula $\mathrm{G}\left(\mathrm{F}_{\mathrm{s}} a \wedge \mathrm{F}_{\mathrm{s}} b\right) \vee \mathrm{G} b$.

In fact, MMAA and $\operatorname{LTL}\left(\mathrm{F}_{\mathrm{s}}, \mathrm{G}_{\mathrm{s}}\right)$ are expressively equivalent. The reverse translation can be found in Appendix $\mathrm{A}$

\section{Translation of MMAA to TGDRA}

In this section we present a translation of an MMAA $\mathcal{A}=\left(S, \Sigma, \delta_{\mathcal{A}}, I, F\right)$ with multitransition relation $\Delta_{\mathcal{A}}$ into an equivalent TGDRA $\mathcal{G}$. At first we build a semiautomaton $\mathcal{T}$ by a double powerset construction (performing dealternation and determinization of the MMAA). Then we describe the transition based generalized Rabin acceptance condition $\mathcal{G R}$ of $\mathcal{G}$.

\subsection{Semiautomaton $\mathcal{T}$}

The idea of our seminautomaton construction is straightforward: a run $\sigma(w)$ of the semiautomaton $\mathcal{T}$ tracks all runs of $\mathcal{A}$ over $w$. More precisely, the state of $\mathcal{T}$ reached after reading a finite input consists of all possible configurations in which $\mathcal{A}$ can be after reading the same input. Hence, states of the semiautomaton are sets of configurations of $\mathcal{A}$ and we call them macrostates. We use $f, s, s_{1}, s_{2}, \ldots$ to denote states of $\mathcal{A}$ ( $f$ stands for an accepting state of $F$ ), $c, c_{1}, c_{2}, \ldots$ to denote configurations of $\mathcal{A}$, and $m, m_{1}, m_{2}, \ldots$ to denote macrostates of $\mathcal{T}$. Further, we use $t, t_{1}, t_{2} \ldots$ to denote the transitions of $\mathcal{A}, T, T_{0}, T_{1} \ldots$ to denote multitransitions of $\mathcal{A}$, and $r, r_{1}, r_{2} \ldots$ to denote the transitions of $\mathcal{T}$, which are called macrotransitions hereafter.

Formally, we define the semiautomaton $\mathcal{T}=\left(M, \Sigma, \delta_{\mathcal{T}}, m_{I}\right)$ for $\mathcal{A}$ as follows:

- $M \subseteq 2^{2^{S}}$ is the set macrostates, restricted to those reachable from the initial macrostate $m_{I}$ by $\delta_{\mathcal{T}}$, 


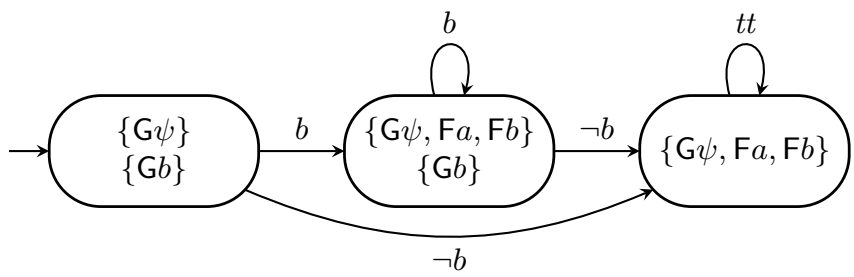

Fig. 2. The semiautomaton $\mathcal{T}$ for the MMAA of Figure $1(\mathrm{a})$

$-\left(m_{1}, \alpha, m_{2}\right) \in \delta_{\mathcal{T}}$ iff $m_{2}=\bigcup_{c \in m_{1}}\left\{c^{\prime} \mid\left(c, \alpha, c^{\prime}\right) \in \Delta_{\mathcal{A}}\right\}$, i.e. for each $m_{1} \in$ $M$ and $\alpha \in \Sigma$, there is a single macrotransition $\left(m_{1}, \alpha, m_{2}\right) \in \delta_{\mathcal{T}}$, where $m_{2}$ consists of target configurations of all $\alpha$-multitransitions leading from configurations in $m_{1}$, and

- $m_{I}=I$ is the initial macrostate.

Figure 2 depicts the semiautomaton $\mathcal{T}$ for the MMAA of Figure 1(a). Each row in a macrostate represents one configuration.

\subsection{Acceptance Condition $\mathcal{G} \mathcal{R}$ of the TGDRA $\mathcal{G}$}

For any subset $Z \subseteq S$, must $(Z)$ denotes the set of must-states of $Z$. An MMAA run $\rho$ is bounded by $Z \subseteq S$ iff $\operatorname{Inf}_{s}(\rho) \subseteq Z$ and $\operatorname{must}\left(\operatorname{Inf}_{s}(\rho)\right)=\operatorname{must}(Z)$. For example, the run of Figure 1(b) is bounded by the set $\{\mathrm{G} \psi, \mathrm{F} a, \mathrm{~F} b\}$.

For any fixed $Z \subseteq S$, we define the set $\mathrm{AC}_{Z} \subseteq 2^{S}$ of allowed configurations of $\mathcal{A}$ and the set $\mathrm{AT}_{Z} \subseteq \delta_{\mathcal{T}}$ of allowed macrotransitions of $\mathcal{T}$ as follows:

$$
\begin{aligned}
\mathrm{AC}_{Z} & =\{c \subseteq Z \mid \text { must }(c)=\operatorname{must}(Z)\} \\
\mathrm{AT}_{Z} & =\left\{\left(m_{1}, \alpha, m_{2}\right) \in \delta_{\mathcal{T}} \mid \exists c_{1} \in \mathrm{AC}_{Z}, c_{2} \in\left(m_{2} \cap \mathrm{AC}_{Z}\right):\left(c_{1}, \alpha, c_{2}\right) \in \Delta_{\mathcal{A}}\right\} \mathbb{1}
\end{aligned}
$$

Clearly, a run $\rho$ of $\mathcal{A}$ is bounded by $Z$ if and only if $\rho$ has a suffix containing only configurations of $\mathrm{AC}_{Z}$. Let $\rho$ be a run over $w$ with such a suffix. As the semiautomaton $\mathcal{T}$ tracks all runs of $\mathcal{A}$ over a given input, the run $\sigma(w)$ of $\mathcal{T}$ 'covers' also $\rho$. Hence, $\sigma(w)$ has a suffix where, for each macrotransition $\left(m_{i}, w_{i}, m_{i+1}\right)$, there exist configurations $c_{1} \in m_{i} \cap \mathrm{AC}_{Z}$ and $c_{2} \in m_{i+1} \cap \mathrm{AC}_{Z}$ satisfying $\left(c_{1}, w_{i}, c_{2}\right) \in \Delta_{\mathcal{A}}$. In other words, $\sigma(w)$ has a suffix containing only macrotransitions of $\mathrm{AT}_{Z}$. This observation is summarized by the following lemma.

Lemma 1. If $\mathcal{A}$ has a run over $w$ bounded by $Z$, then the run $\sigma(w)$ of $\mathcal{T}$ contains a suffix of macrotransitions of $\mathrm{AT}_{Z}$.

In fact, the other direction can be proved as well: if $\sigma(w)$ contains a suffix of macrotransitions of $\mathrm{AT}_{Z}$, then $\mathcal{A}$ has a run over $w$ bounded by $Z$.

For each $f \in F \cap Z$, we also define the set $\mathrm{AT}_{Z}^{f}$ as the set of all macrotransitions in $\mathrm{AT}_{Z}$ such that $\mathcal{A}$ contains a non-looping transition of $f$ with the same label and with the target configuration not leaving $Z$ :

$$
\operatorname{AT}_{Z}^{f}=\left\{\left(m_{1}, \alpha, m_{2}\right) \in \operatorname{AT}_{Z} \mid \exists(f, \alpha, c) \in \delta_{\mathcal{A}}: f \notin c, c \subseteq Z\right\}
$$

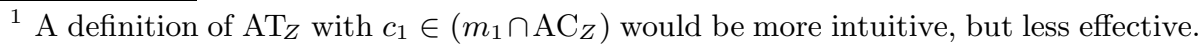


Using the sets $\mathrm{AT}_{Z}$ and $\mathrm{AT}_{Z}^{f}$, we define one generalized Rabin pair $\mathcal{G} \mathcal{R}_{Z}$ for each subset of states $Z \subseteq S$ :

$$
\mathcal{G} \mathcal{R}_{Z}=\left(\delta_{\mathcal{T}} \backslash \mathrm{AT}_{Z},\left\{\mathrm{AT}_{Z}^{f}\right\}_{f \in F \cap Z}\right)
$$

Lemma 2. If there is an accepting run $\rho$ of $\mathcal{A}$ over $w$ then the run $\sigma(w)$ of $\mathcal{T}$ satisfies $\mathcal{G R}_{Z}$ for $Z=\operatorname{Inf}_{s}(\rho)$.

Proof. As $\rho$ is bounded by $Z$, Lemma 1 implies that $\sigma(w)$ has a suffix $r_{i} r_{i+1} \ldots$ of macrotransitions of $\mathrm{AT}_{Z}$. Thus $\operatorname{Inf}_{t}(\sigma(w)) \cap\left(\delta_{\mathcal{T}} \backslash \mathrm{AT}_{Z}\right)=\emptyset$.

As $Z=\operatorname{Inf}_{s}(\rho)$ and $\rho=T_{0} T_{1} \ldots$ is accepting, for each $f \in F \cap Z, \rho$ includes infinitely many multitransitions $T_{j}$ where $f \in \operatorname{dom}\left(T_{j}\right)$ and $T_{j}$ contains a non-looping transition $\left(f, w_{j}, c\right) \in \delta_{\mathcal{A}}$ satisfying $f \notin c$ and $c \subseteq Z$. Hence, the corresponding macrotransitions $r_{j}$ that are also in the mentioned suffix $r_{i} r_{i+1} \ldots$ of $\sigma(w)$ are elements of $\operatorname{AT}_{Z}^{f}$. Therefore, $\operatorname{Inf}_{t}(\sigma(w)) \cap \mathrm{AT}_{Z}^{f} \neq \emptyset$ for each $f \in F \cap Z$ and $\sigma(w)$ satisfies $\mathcal{G R}_{Z}$.

Lemma 3. If a run $\sigma(w)$ of $\mathcal{T}$ satisfies $\mathcal{G R}_{Z}$ then there is an accepting run of $\mathcal{A}$ over $w$ bounded by $Z$.

Proof. Let $\sigma(w)=r_{0} r_{1} \ldots$ be a run of $\mathcal{T}$ satisfying $\mathcal{G R}_{Z}$, i.e. $\sigma(w)$ has a suffix of macrotransitions of $\mathrm{AT}_{Z}$ and $\sigma(w)$ contains infinitely many macrotransitions of $\mathrm{AT}_{Z}^{f}$ for each $f \in F \cap Z$. Let $r_{i}=\left(m_{i}, w_{i}, m_{i+1}\right)$ be the first macrotransition of the suffix. The definition of $\mathrm{AT}_{Z}$ implies that there is a configuration $c \in$ $m_{i+1} \cap \mathrm{AC}_{Z}$. The construction of $\mathcal{T}$ guarantees that there exists a sequence of multitransitions of $\mathcal{A}$ leading to the configuration $c$. More precisely, there is a sequence $T_{0} T_{1} \ldots T_{i}$ such that $\operatorname{dom}\left(T_{0}\right)$ is an initial configuration of $\mathcal{A}, T_{j}$ is labelled by $w_{j}$ for each $0 \leq j \leq i$, range $\left(T_{j}\right)=\operatorname{dom}\left(T_{j+1}\right)$ for each $0 \leq j<i$, and range $\left(T_{i}\right)=c$. We show that this sequence is in fact a prefix of an accepting run of $\mathcal{A}$ over $w$ bounded by $Z$.

We inductively define a multitransition sequence $T_{i+1} T_{i+2} \ldots$ completing this run. The definition uses the suffix $r_{i+1} r_{i+2} \ldots$ of $\sigma(w)$. Let us assume that $j>i$ and that range $\left(T_{j-1}\right)$ is a configuration of $\mathrm{AC}_{Z}$. We define $T_{j}$ to contain one $w_{j}$ transition of $s$ for each $s \in \operatorname{range}\left(T_{j-1}\right)$. Thus we get $\operatorname{dom}\left(T_{j}\right)=\operatorname{range}\left(T_{j-1}\right)$. As $r_{j} \in \mathrm{AT}_{Z}$, there exists a multitransition $T^{\prime}$ labelled by $w_{j}$ such that both source and target configurations of $T^{\prime}$ are in $\mathrm{AC}_{Z}$. For each must-state $s \in \operatorname{range}\left(T_{j-1}\right)$, $T_{j}$ contains the same transition leading from $s$ as contained in $T^{\prime}$. For maystates $f \in \operatorname{range}\left(T_{j-1}\right)$, we have two cases. If $r_{j} \in \mathrm{AT}_{Z}^{f}, T_{j}$ contains a nonlooping transition leading from $f$ to some states in $Z$. The existence of such a transition follows from the definition of $\mathrm{AT}_{Z}^{f}$. For the remaining may-states, $T_{j}$ uses selfloops. Formally, $T_{j}=\left\{t_{j}^{s} \mid s \in \operatorname{range}\left(T_{j-1}\right)\right\}$, where

$$
t_{j}^{s}= \begin{cases}\left(s, w_{j}, c_{s}\right) \text { contained in } T^{\prime} & \text { if } s \in \operatorname{must}(Z) \\ \left(s, w_{j},\{s\}\right) & \text { if } s \in F \wedge r_{j} \notin \mathrm{AT}_{Z}^{s} \\ \left(s, w_{j}, c_{s}\right) \text { where } c_{s} \subseteq Z, s \notin c_{s} & \text { if } s \in F \wedge r_{j} \in \mathrm{AT}_{Z}^{s}\end{cases}
$$

One can easily check that $\operatorname{range}\left(T_{j}\right) \in \mathrm{AC}_{Z}$ and we continue by building $T_{j+1}$. 
To sum up, the constructed run is bounded by $Z$. Moreover, $T_{j}$ contains no looping transition of $f$ whenever $r_{j} \in \mathrm{AT}_{Z}^{f}$. As the run $\sigma(w)$ is accepting, $r_{j} \in \mathrm{AT}_{Z}^{f}$ holds infinitely often for each $f \in F \cap Z$. The constructed run of $\mathcal{A}$ over $w$ is thus accepting.

The previous two lemmata give us the following theorem.

Theorem 1. The $T G D R A \mathcal{G}=\left(\mathcal{T},\left\{\mathcal{G R}_{Z} \mid Z \subseteq S\right\}\right)$ describes the same language as $\mathcal{A}$.

\section{Translation of TGDRA to DRA}

This section presents a variant of the standard degeneralization procedure. At first we illustrate the idea on a TGDRA $\mathcal{G}^{\prime}=\left(M, \Sigma, \delta_{\mathcal{T}}, m_{I},\left\{\left(K,\left\{L^{j}\right\}_{1 \leq j \leq h}\right)\right\}\right)$ with one generalized Rabin pair. Recall that a run is accepting if it has a suffix not using macrotransitions of $K$ and using macrotransitions of each $L^{j}$ infinitely often.

An equivalent DRA $\mathcal{D}^{\prime}$ consists of $h+2$ copies of $\mathcal{G}^{\prime}$. The copies are called levels. We start at the level 1. Intuitively, being at a level $j$ for $1 \leq j \leq h$ means that we are waiting for a transition from $L^{j}$. Whenever a transition of $K$ appears, we move to the level 0 . A transition $r \notin K$ gets us from a level $j$ to the maximal level $l \geq j$ such that $r \in L^{j^{\prime}}$ for each $j \leq j^{\prime}<l$. The levels 0 and $h+1$ have the same transitions (including target levels) as the level 1. A run of $\mathcal{G}^{\prime}$ is accepting if and only if the corresponding run of $\mathcal{D}^{\prime}$ visits the level 0 only finitely often and it visits the level $h+1$ infinitely often.

In the general case, we track the levels for all generalized Rabin pairs simultaneously. Given a TGDRA $\mathcal{G}=\left(M, \Sigma, \delta_{\mathcal{T}}, m_{I},\left\{\left(K_{i},\left\{L_{i}^{j}\right\}_{1 \leq j \leq h_{i}}\right)\right\}_{1 \leq i \leq k}\right)$, we construct an equivalent DRA as $\mathcal{D}=\left(Q, \Sigma, \delta_{\mathcal{D}}, q_{i},\left\{\left(K_{i}^{\prime}, L_{i}^{\prime}\right)\right\}_{1 \leq i \leq k}\right)$, where

- $Q=M \times\left\{0,1, \ldots, h_{1}+1\right\} \times \cdots \times\left\{0,1, \ldots, h_{k}+1\right\}$,

$-\left(\left(m, l_{1}, \ldots, l_{k}\right), \alpha,\left(m^{\prime}, l_{1}^{\prime}, \ldots, l_{k}^{\prime}\right)\right) \in \delta_{\mathcal{D}}$ iff $r=\left(m, \alpha, m^{\prime}\right) \in \delta_{\mathcal{T}}$ and for each $1 \leq i \leq k$ it holds

$$
l_{i}^{\prime}= \begin{cases}0 & \text { if } r \in K_{i} \\ \max \left\{l_{i} \leq l \leq h_{i}+1 \mid \forall l_{i} \leq j<l: r \in L_{i}^{j}\right\} & \text { if } r \notin K_{i} \wedge 1 \leq l_{i} \leq h_{i} \\ \max \left\{1 \leq l \leq h_{i}+1 \mid \forall 1 \leq j<l: r \in L_{i}^{j}\right\} & \text { if } r \notin K_{i} \wedge l_{i} \in\left\{0, h_{i}+1\right\},\end{cases}
$$

$-q_{i}=\left(m_{I}, 1, \ldots, 1\right)$,

- $K_{i}^{\prime}=\left\{\left(m, l_{1}, \ldots, l_{k}\right) \in Q \mid l_{i}=0\right\}$, and

- $L_{i}^{\prime}=\left\{\left(m, l_{1}, \ldots, l_{k}\right) \in Q \mid l_{i}=h_{i}+1\right\}$.

\section{Complexity}

This section discusses the upper bounds of the individual steps of our translation and compares the overall complexity to the complexity of the other translations. 
Given a formula $\varphi$ of $\operatorname{LTL}\left(\mathrm{F}_{\mathrm{s}}, \mathrm{G}_{\mathrm{s}}\right)$, we produce an MMAA with at most $n$ states, where $n$ is the length of $\varphi$. Then we build the TGDRA $\mathcal{G}$ with at most $2^{2^{n}}$ states and at most $2^{n}$ generalized Rabin pairs. To obtain the DRA $\mathcal{D}$, we multiply the state space by at most $|Z|+2$ for each generalized Rabin pair $\mathcal{G R}_{Z}$. The value of $|Z|$ is bounded by $n$. Altogether, we can derive an upper bound on the number of states of the resulting DRA as

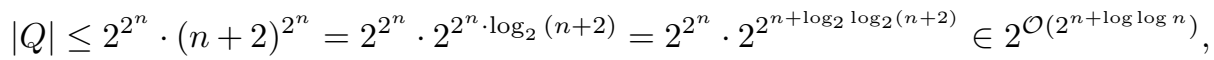

which is the same bound as in [19], but lower than $2^{\mathcal{O}\left(2^{n+\log n}\right)}$ of 1tl2dstar. It is worth mentioning that the number of states of our TGDRA is bounded by $2^{2^{|\varphi|}}$ while the number of states of the GDRA produced by Rabinizer is bounded by $2^{2^{|\varphi|}} \cdot 2^{A P(\varphi)}$.

\section{Simplifications and Translation Improvements}

An important aspect of our translation process is simplification of all intermediate results leading to smaller resulting DRA.

We simplify input formulae by reduction rules of LTL3BA, see [3] for more details. Additionally, we rewrite the subformulae of the form GF $\psi$ and $\mathrm{FG} \psi$ to equivalent formulae $\mathrm{GF}_{\mathrm{s}} \psi$ and $\mathrm{FG}_{\mathrm{s}} \psi$ respectively. This preference of strict temporal operators often yields smaller resulting automata.

Alternating automata are simplified in the same way as in LTL2BA: removing unreachable states, merging equivalent states, and removing redundant transitions, see [14] for details.

We improve the translation of an MMAA $\mathcal{A}$ to a TGDRA $\mathcal{G}$ in order to reduce the number of generalized Rabin pairs of $\mathcal{G}$. One can observe that, for any accepting run $\rho$ of $\mathcal{A}, \operatorname{Inf}_{s}(\rho)$ contains only states reachable from some muststate. Hence, in the construction of acceptance condition of $\mathcal{G}$ we can consider only subsets $Z$ of states of $\mathcal{A}$ of this form. Further, we omit a subset $Z$ if, for each accepting run over $w$ bounded by $Z$, there is also an accepting run over $w$ bounded by some $Z^{\prime} \subseteq Z$. The formal description of subsets $Z$ considered in the construction of the TGDRA $\mathcal{G}$ is described in Appendix B.

If a run $T_{0} T_{1} \ldots$ of an MMAA satisfies range $\left(T_{i}\right)=\emptyset$ for some $i$, then $T_{j}=\emptyset$ for all $j \geq i$ and the run is accepting. We use this observation to improve the construction of the semiautomaton $\mathcal{T}$ of the TGDRA $\mathcal{G}$ : if a macrostate $m$ contains the empty configuration, we remove all other configurations from $m$.

After we build the TGDRA, we simplify its acceptance condition in three ways (similar optimizations are also performed by Rabinizer).

1. We remove some generalized Rabin pairs $\left(K_{i},\left\{L_{i}^{j}\right\}_{j \in J_{i}}\right)$ that cannot be satisfied by any run, in particular when $K_{i}=\delta_{\mathcal{T}}$ or $L_{i}^{j}=\emptyset$ for some $j \in J_{i}$.

2. We remove $L_{i}^{j}$ if there is some $l \in J_{i}$ such that $L_{i}^{l} \subseteq L_{i}^{j}$.

3. If the fact that a run $\rho$ satisfies the pair $\mathcal{G R}_{Z}$ implies that $\rho$ satisfies also some other pair $\mathcal{G R}_{Z^{\prime}}$, we remove $\mathcal{G} \mathcal{R}_{Z}$. 
Finally, we simplify the state spaces of both TGDRA and DRA such that we iteratively merge the equivalent states. Two states of a DRA $\mathcal{D}$ are equivalent if they belong to the same sets of the acceptance condition of $\mathcal{D}$ and, for each $\alpha$, their $\alpha$-transitions lead to the same state. Two states of a TGDRA $\mathcal{G}$ are equivalent if, for each $\alpha$, their $\alpha$-transitions lead to the same state and belong to the same sets of the acceptance condition of $\mathcal{G}$. Moreover, if the initial state of $\mathcal{D}$ or $\mathcal{G}$ has no selfloop, we check its equivalence to another state regardless of the acceptance condition (note that a membership in acceptance condition sets is irrelevant for states or transitions that are passed at most once by any run).

Of course, we consider only the reachable state space at every step.

\section{Beyond $\operatorname{LTL}\left(\mathbf{F}_{s}, \mathbf{G}_{s}\right)$ Fragment: May/Must in the Limit}

The Section 4 shows a translation of MMAA into TGDRA. In fact, our translation can be used for a larger class of very weak alternating automata called may/must in the limit automata (limMMAA). A VWAA $\mathcal{B}$ is a limMMAA if $\mathcal{B}$ contains only must-states, states without looping transitions, and co-Büchi accepting states (not exclusively may-states), and each state reachable from a must-state is either a must- or a may-state. Note that each accepting run of a limMMAA has a suffix that contains either only empty configurations, or configurations consisting of must-states and may-states reachable from must-states. Hence, the MMAA to TGDRA translation produces correct results also for limMMAA under an additional condition: generalized Rabin pairs $\mathcal{G R}_{Z}$ are constructed only for sets $Z$ that contain only must-states and may-states reachable from them.

We can obtain limMMAA by the LTL to VWAA translation of [14 when it is applied to an LTL fragment defined as

$$
\varphi::=\psi|\varphi \vee \varphi| \varphi \wedge \varphi|\mathrm{X} \varphi| \varphi \mathrm{U} \varphi,
$$

where $\psi$ ranges over $\operatorname{LTL}\left(\mathrm{F}_{\mathbf{s}}, \mathrm{G}_{\mathrm{s}}\right)$. Note that this fragment is strictly more expressive than $\operatorname{LTL}\left(\mathrm{F}_{\mathrm{s}}, \mathrm{G}_{\mathrm{s}}\right)$.

\section{$9 \quad$ Experimental Results}

We have made an experimental implementation of our translation (referred to as $L T L 3 D R A$ ). The translation of LTL to alternating automata is taken from LTL3BA [3. We compare the automata produced by LTL3DRA to those produced by Rabinizer and ltl2dstar. All the experiments are run on a Linux laptop (2.4GHz Intel Core i7, 8GB of RAM) with a timeout set to 5 minutes.

Tables given below (i) compare the sizes of the DRA produced by all the tools and (ii) show the number of states of the generalized automata produced by LTL3DRA and Rabinizer. Note that LTL3DRA uses TGDRA whereas Rabinizer uses (state-based) GDRA, hence the numbers of their states cannot be directly compared. The sizes of DRA are written as $s(r)$, where $s$ is the number of states 


\begin{tabular}{|c|c|c|c|c|c|c|}
\hline \multirow{2}{*}{\multicolumn{2}{|c|}{ Formula }} & \multicolumn{2}{|c|}{ LTL3DRA } & \multicolumn{2}{|c|}{ Rabinizer } & \multirow{2}{*}{$\frac{\text { ltl2dstar }}{\text { DRA }}$} \\
\hline & & $\overline{D R A}$ & TGDRA & $\overline{D R A}$ G & $\overline{\mathrm{DRA}}$ & \\
\hline \multicolumn{2}{|c|}{$\mathrm{G}(a \vee \mathrm{F} b)$} & $3(2)$ & 2 & $4(2)$ & 5 & $4(1)$ \\
\hline \multicolumn{2}{|c|}{$\mathrm{FG} a \vee \mathrm{FG} b \vee \mathrm{GF} c$} & $8(3)$ & 1 & $8(3)$ & 8 & $8(3)$ \\
\hline \multicolumn{2}{|c|}{$\mathrm{F}(a \vee b)$} & $2(1)$ & 2 & $2(1)$ & 2 & $2(1)$ \\
\hline \multicolumn{2}{|c|}{$\mathrm{GF}(a \vee b)$} & $2(1)$ & 1 & $2(1)$ & 4 & $2(1)$ \\
\hline \multicolumn{2}{|c|}{$\mathrm{G}(a \vee \mathrm{F} a)$} & $2(1)$ & 1 & $2(2)$ & 2 & $2(1)$ \\
\hline \multicolumn{2}{|c|}{$\mathrm{G}(a \vee b \vee c)$} & $2(1)$ & 2 & $2(1)$ & 8 & $3(1)$ \\
\hline \multicolumn{2}{|c|}{$\mathrm{G}(a \vee \mathrm{F}(b \vee c))$} & $3(2)$ & 2 & $4(2)$ & 9 & $4(1)$ \\
\hline \multicolumn{2}{|c|}{$\mathrm{F} a \vee \mathrm{G} b$} & $3(2)$ & 3 & $3(2)$ & 3 & $4(2)$ \\
\hline \multicolumn{2}{|c|}{$\mathrm{G}(a \vee \mathrm{F}(b \wedge c))$} & $3(2)$ & 2 & $4(2)$ & 11 & $4(1)$ \\
\hline \multicolumn{2}{|c|}{$\mathrm{FG} a \vee \mathrm{GF} b$} & $4(2)$ & 1 & $4(2)$ & 4 & $4(2)$ \\
\hline \multicolumn{2}{|c|}{$\mathrm{GF}(a \vee b) \wedge \mathrm{GF}(b \vee c)$} & $3(1)$ & 1 & $3(1)$ & 8 & $7(2)$ \\
\hline \multicolumn{2}{|c|}{$(\mathrm{FF} a \wedge \mathrm{G} \neg a) \vee(\mathrm{GG} \neg a \wedge \mathrm{F} a)$} & $1(0)$ & 1 & $1(0)$ & 1 & $1(0)$ \\
\hline \multicolumn{2}{|c|}{$\mathrm{GF} a \wedge \mathrm{FG} b$} & $3(1)$ & 1 & $3(1)$ & 4 & $3(1)$ \\
\hline \multicolumn{2}{|c|}{$(\mathrm{GF} a \wedge \mathrm{FG} b) \vee(\mathrm{FG} \neg a \wedge \mathrm{GF} \neg b)$} & $4(2)$ & 1 & $4(2)$ & 4 & $5(2)$ \\
\hline \multicolumn{2}{|c|}{$\mathrm{FG} a \wedge \mathrm{GF} a$} & $2(1)$ & 1 & $2(1)$ & 2 & $2(1)$ \\
\hline \multicolumn{2}{|c|}{$\mathrm{G}(\mathrm{F} a \wedge \mathrm{F} b)$} & $3(1)$ & 1 & $3(1)$ & 4 & $5(1)$ \\
\hline \multicolumn{2}{|c|}{$\mathrm{F} a \wedge \mathrm{F} \neg a$} & $4(1)$ & 4 & $4(1)$ & 4 & $4(1)$ \\
\hline \multirow{2}{*}{\multicolumn{2}{|c|}{$\begin{array}{l}(\mathrm{G}(b \vee \mathrm{GF} a) \wedge \mathrm{G}(c \vee \mathrm{GF} \neg a)) \vee \mathrm{G} b \vee \mathrm{G} c \\
(\mathrm{G}(b \vee \mathrm{FG} a) \wedge \mathrm{G}(c \vee \mathrm{FG} \neg a)) \vee \mathrm{G} b \vee \mathrm{G} c\end{array}$}} & $12(3)$ & 4 & $18(4)$ & 18 & $13(3)$ \\
\hline & & $4(2)$ & 4 & $6(3)$ & 18 & $14(4)$ \\
\hline \multirow{3}{*}{\multicolumn{2}{|c|}{$\begin{array}{c}(\mathrm{F}(b \wedge \mathrm{FG} a) \vee \mathrm{F}(c \wedge \mathrm{FG} \neg a)) \wedge \mathrm{F} b \wedge \mathrm{F} c \\
(\mathrm{~F}(b \wedge \mathrm{GF} a) \vee \mathrm{F}(c \wedge \mathrm{GF} \neg a)) \wedge \mathrm{F} b \wedge \mathrm{F} c \\
\mathrm{GF}(\mathrm{F} a \vee \mathrm{GF} b \vee \mathrm{FG}(a \vee b))\end{array}$}} & $5(2)$ & 4 & $5(2)$ & 18 & $7(1)$ \\
\hline & & $5(2)$ & 4 & $5(2)$ & 18 & $7(2)$ \\
\hline & & $4(3)$ & 1 & $4(3)$ & 4 & $14(4)$ \\
\hline \multicolumn{2}{|c|}{$\mathrm{FG}(\mathrm{F} a \vee \mathrm{GF} b \vee \mathrm{FG}(a \vee b))$} & $4(3)$ & 1 & $4(3)$ & 4 & $145(9)$ \\
\hline \multicolumn{2}{|c|}{$\mathrm{FG}(\mathrm{F} a \vee \mathrm{GF} b \vee \mathrm{FG}(a \vee b) \vee \mathrm{FG} b)$} & $4(3)$ & 1 & $4(3)$ & 4 & $145(9)$ \\
\hline \multirow{4}{*}{$\bigwedge_{i=1}^{n}\left(\mathrm{GF} a_{i} \rightarrow \mathrm{GF} b_{i}\right)$} & $n=1$ & $4(2)$ & 1 & $4(2)$ & 4 & $4(2)$ \\
\hline & $n=2$ & $18(4)$ & 1 & $20(4)$ & 16 & $11324(8)$ \\
\hline & $n=3$ & $166(8)$ & 1 & $470(8)$ & 64 & timeout \\
\hline & $n=4$ & $7408(16)$ & 1 & timeo & & timeout \\
\hline \multirow{6}{*}{$\bigwedge_{i=1}^{n}\left(\mathrm{GF} a_{i} \vee \mathrm{FG} a_{i+1}\right)$} & $n=1$ & $4(2)$ & 1 & $4(2)$ & 4 & $4(2)$ \\
\hline & $n=2$ & $10(4)$ & 1 & $11(4)$ & 8 & $572(7)$ \\
\hline & $n=3$ & $36(6)$ & 1 & $52(6)$ & 16 & $290046(13)$ \\
\hline & $n=4$ & $178(9)$ & 1 & $1288(9)$ & 32 & timeout \\
\hline & $n=5$ & $1430(14)$ & 1 & timeo & & timeout \\
\hline & $n=6$ & $20337(22)$ & 1 & timeo & & timeout \\
\hline
\end{tabular}

Table 1. The benchmark from [13] extended by one parametric formula.

and $r$ is the number of Rabin pairs. For each formula, the size of the smallest DRA (measured by the number of states and, in the case of equality, by the number of Rabin pairs) is printed in bold.

Table 1 shows the results on formulae from [13] extended with another parametric formula. For the two parametric formulae, we give all the parameter values $n$ for which at least one tool finished before timeout. For all formulae in the table, our experimental implementation generates automata of the same 


\begin{tabular}{|c|c|c|c|c|c|}
\hline & \multicolumn{2}{|c|}{ LTL3DRA } & \multicolumn{2}{|c|}{ Rabinizer } & \multirow{2}{*}{\begin{tabular}{|c|} 
1tl2dstar \\
DRA
\end{tabular}} \\
\hline & DRA & TGDRA & DRA & $\overline{\text { GDRA }}$ & \\
\hline$\varphi_{2}$ & $4(2)$ & 4 & & & $5(2)$ \\
\hline$\varphi_{3}$ & $4(2)$ & 3 & $4(2)$ & 5 & $4(1)$ \\
\hline$\varphi_{7}$ & $4(2)$ & 3 & - & & $4(2)$ \\
\hline$\varphi_{8}$ & $3(2)$ & 3 & $3(2)$ & 5 & $4(2)$ \\
\hline$\varphi_{11}$ & $6(2)$ & 6 & & & $10(3)$ \\
\hline$\varphi_{12}$ & $8(2)$ & 8 & & & $9(2)$ \\
\hline$\varphi_{13}$ & $7(3)$ & 7 & & & $11(3)$ \\
\hline$\varphi_{17}$ & $4(2)$ & 4 & - & & $5(2)$ \\
\hline$\varphi_{18}$ & $4(2)$ & 3 & $4(2)$ & 5 & $4(1)$ \\
\hline$\varphi_{21}$ & $4(2)$ & 3 & & & $4(2)$ \\
\hline$\varphi_{22}$ & $4(2)$ & 4 & & & $5(2)$ \\
\hline$\varphi_{23}$ & $5(3)$ & 4 & - & & $5(3)$ \\
\hline$\varphi_{26}$ & $3(2)$ & 2 & $4(2)$ & 5 & $4(1)$ \\
\hline
\end{tabular}

\begin{tabular}{|c|c|c|c|c|c|}
\hline & \multicolumn{2}{|c|}{ LTL3DRA } & \multicolumn{2}{|c|}{ Rabinizer } & 1 tl2dstar \\
\hline & DRA & TGDRA & DRA & $\overline{\text { GDRA }}$ & DRA \\
\hline & $4(2)$ & 4 & 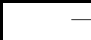 & - & $5(2)$ \\
\hline$\varphi$ & $6(3)$ & 3 & $8(3)$ & 14 & $5(1)$ \\
\hline & $4(2)$ & 4 & - & - & $6(2)$ \\
\hline & $5(2)$ & 5 & - & & $7(2)$ \\
\hline$\varphi$ & $5(2)$ & 5 & - & & $7(3)$ \\
\hline & $6(3)$ & 4 & - & & $6(2)$ \\
\hline & $6(2)$ & 6 & - & & $8(3)$ \\
\hline & $7(4)$ & 5 & - & & $6(3)$ \\
\hline$\varphi_{41}$ & $21(3)$ & 7 & - & & $45(3)$ \\
\hline$\varphi_{42}$ & $12(2)$ & 12 & - & & $17(2)$ \\
\hline$\varphi$ & $15(3)$ & 5 & - & & $20(2)$ \\
\hline$\varphi_{47}$ & $7(2)$ & 7 & - & & $6(2)$ \\
\hline$\varphi$ & $14(3)$ & 6 & 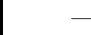 & & $24(2)$ \\
\hline & $7(2)$ & 7 & - & - & $6(2)$ \\
\hline
\end{tabular}

Table 2. The benchmark with selected formulae from SPEC PATTERNS. $\varphi_{i}$ denotes the $i$-th formula on the web page.

or smaller size as the others. Especially in the case of parametric formulae, the automata produced by LTL3DRA are considerably smaller. We also note that the TGDRA constructed for the formulae are typically very small.

Table 2 shows the results on formulae from Spec PAtTerns [12] (available onlin(2). We only take formulae LTL3DRA is able to work with, i.e. the formulae of the LTL fragment defined in Section 8. The fragment covers 27 out of 55 formulae listed on the web page. The dash sign in Rabinizer's column means that Rabinizer cannot handle the corresponding formula as it is not from the $\operatorname{LTL}(F, G)$ fragment. For most of the formulae in the table, LTL3DRA produces the smallest DRA. In the remaining cases, the DRA produced by our translation is only slightly bigger than the smallest one. The table also illustrates that LTL3DRA handles many (pseudo)realistic formulae not included in LTL $(F, G)$. Four more parametric benchmarks are provided in Appendix $\mathrm{C}$

\section{Conclusion}

We present another Safraless translation of an LTL fragment to deterministic Rabin automata (DRA). Our translation employs a new class of may/must alternating automata. We prove that the class is expressively equivalent to the $\operatorname{LTL}\left(F_{s}, G_{s}\right)$ fragment. Experimental results show that our translation typically produces DRA of a smaller or equal size as the other two translators of LTL (i.e. Rabinizer and ltl2dstar) and it sometimes produces automata that are significantly smaller.

\footnotetext{
${ }^{2}$ http://patterns.projects.cis.ksu.edu/documentation/patterns/ltl.shtml
} 


\section{References}

1. R. Alur and S. L. Torre. Deterministic generators and games for LTL fragments. ACM Trans. Comput. Log., 5(1):1-25, 2004.

2. T. Babiak, T. Badie, A. Duret-Lutz, M. Křetínský, and J. Strejček. Compositional approach to suspension and other improvements to LTL translation. In SPIN 2013, volume 7976 of $L N C S$, pages 81-98. Springer, 2013.

3. T. Babiak, M. Křetínský, V. Řehák, and J. Strejček. LTL to Büchi automata translation: Fast and more deterministic. In TACAS 2012, volume 7214 of LNCS, pages 95-109. Springer, 2012.

4. C. Baier and J.-P. Katoen. Principles of Model Checking. MIT Press, 2008.

5. K. Chatterjee, A. Gaiser, and J. Křetínský. Automata with generalized Rabin pairs for probabilistic model checking and LTL synthesis. In CAV 2013, volume 8044 of $L N C S$, pages 559-575. Springer, 2013.

6. A. Church. Logic, arithmetic, and automata. In Proceedings of the International Congress of Mathematicians, pages 23-35. Institut Mittag-Leffler, 1962.

7. E. M. Clarke, O. Grumberg, and D. A. Peled. Model Checking. MIT Press, 1999.

8. C. Courcoubetis and M. Yannakakis. The complexity of probabilistic verification. J. $A C M, 42(4): 857-907,1995$.

9. J.-M. Couvreur. On-the-fly verification of temporal logic. In FM 1999, volume 1708 of LNCS, pages 253-271. Springer, 1999.

10. M. Daniele, F. Giunchiglia, and M. Y. Vardi. Improved automata generation for linear temporal logic. In $C A V$ 1999, volume 1633 of $L N C S$, pages 249-260. Springer, 1999.

11. A. Duret-Lutz. LTL translation improvements in Spot. In VECoS 2011, Electronic Workshops in Computing. British Computer Society, 2011.

12. M. B. Dwyer, G. S. Avrunin, and J. C. Corbett. Patterns in property specifications for finite-state verification. In ICSE 1999, pages 411-420. IEEE, 1999.

13. A. Gaiser, J. Křetínský, and J. Esparza. Rabinizer: Small deterministic automata for $\operatorname{LTL}(\mathrm{F}, \mathrm{G})$. In ATVA 2012, volume 7561 of $L N C S$, pages 72-76, 2012.

14. P. Gastin and D. Oddoux. Fast LTL to Büchi Automata Translation. In $C A V$ 2001, volume 2102 of LNCS, pages 53-65. Springer, 2001.

15. J. Geldenhuys and H. Hansen. Larger automata and less work for LTL model checking. In SPIN 2006, volume 3925 of $L N C S$, pages 53-70. Springer, 2006.

16. J. Klein. ltl2dstar - LTL to deterministic Streett and Rabin automata. http://www.ltl2dstar.de.

17. J. Klein and C. Baier. Experiments with deterministic $\omega$-automata for formulas of linear temporal logic. Theor. Comput. Sci., 363(2):182-195, 2006.

18. J. Klein and C. Baier. On-the-fly stuttering in the construction of deterministic w-automata. In CIAA 2007, volume 4783 of $L N C S$, pages 51-61. Springer, 2007.

19. J. Křetínský and J. Esparza. Deterministic automata for the (F, G)-fragment of LTL. In $C A V$ 2012, volume 7358 of $L N C S$, pages 7-22. Springer, 2012.

20. O. Kupferman. Recent challenges and ideas in temporal synthesis. In SOFSEM 2012, volume 7147 of $L N C S$, pages 88-98. Springer, 2012.

21. M. Kwiatkowska, G. Norman, and D. Parker. PRISM 4.0: Verification of probabilistic real-time systems. In $C A V$ 2011, volume 6806 of $L N C S$, pages 585-591. Springer, 2011.

22. A. Morgenstern and K. Schneider. From LTL to symbolically represented deterministic automata. In VMCAI 2008, volume 4905 of $L N C S$, pages 279-293. Springer, 2008 . 
23. N. Piterman. From nondeterministic Büchi and Streett automata to deterministic parity automata. Logical Methods in Computer Science, 3(3), 2007.

24. N. Piterman, A. Pnueli, and Y. Sa'ar. Synthesis of reactive(1) designs. In VMCAI 2006, volume 3855 of $L N C S$, pages 364-380. Springer, 2006.

25. A. Pnueli. The temporal logic of programs. In FOCS 1977, pages 46-57. IEEE, 1977.

26. A. Pnueli and R. Rosner. On the synthesis of an asynchronous reactive module. In ICALP 1989, volume 372 of $L N C S$, pages 652-671. Springer, 1989.

27. S. Safra. On the complexity of omega-automata. In FOCS 1988, pages 319-327. IEEE Computer Society, 1988.

28. S. Schewe. Tighter bounds for the determinisation of Büchi automata. In FOSSACS 2009, volume 5504 of $L N C S$, pages 167-181. Springer, 2009.

29. F. Somenzi and R. Bloem. Efficient Büchi automata from LTL formulae. In $C A V$ 2000, volume 1855 of LNCS, pages 248-263. Springer, 2000.

30. M. Y. Vardi. Automatic verification of probabilistic concurrent finite-state programs. In FOCS 1985, pages 327-338. IEEE Computer Society, 1985.

31. M. Y. Vardi and P. Wolper. An automata-theoretic approach to automatic program verification. In LICS 1986, pages 332-344. IEEE Computer Society, 1986. 


\section{A Translation of MMAA to $\operatorname{LTL}\left(\mathrm{F}_{\mathrm{s}}, \mathrm{G}_{\mathrm{s}}\right)$}

We can assume that may-states have no looping transitions except selfloops. Indeed, any application of a looping transition that is not a selfloop can be always replaced by an application of a selfloop with the same label. This change is safe as it cannot transform an accepting run into a non-accepting one.

Let $\mathcal{A}=\left(Q, 2^{A P^{\prime}}, \delta, I, F\right)$ be an MMAA. For any $\alpha \in 2^{A P^{\prime}}$, we define $\psi_{\alpha}$ to be a formula satisfied exactly by the words starting with $\alpha$ :

$$
\psi_{\alpha}=\left(\bigwedge_{a \in \alpha} a\right) \wedge\left(\bigwedge_{a \in A P^{\prime} \backslash \alpha} \neg a\right)
$$

Now we inductively define a formula $\varphi_{s}$ for each $s \in Q$. The formula $\varphi_{s}$ is satisfied by any word for which there is an accepting run of $\mathcal{A}$ starting in the configuration $\{s\}$. Admissibility of the inductive definition follows from the fact that $\mathcal{A}$ is a very weak automaton, i.e. there is a partial order on $Q$ such that transitions of a state $s$ can lead only to $s$ or lower states.

$$
\varphi_{s}= \begin{cases}\mathrm{F} \bigvee_{(s, \alpha, c) \in \delta, c \neq\{s\}}\left(\psi_{\alpha} \wedge \bigwedge_{q \in c} \mathrm{X} \varphi_{q}\right) & \text { if } s \text { is a may-state } \\ \mathrm{G} \bigvee_{(s, \alpha, c) \in \delta}\left(\psi_{\alpha} \wedge \bigwedge_{q \in c \backslash\{s\}} \mathrm{X} \varphi_{q}\right) & \text { if } s \text { is a must-state } \\ \bigvee_{(s, \alpha, c) \in \delta}\left(\psi_{\alpha} \wedge \bigwedge_{q \in c} \mathrm{X} \varphi_{q}\right) & \text { if } s \text { is a loopless state }\end{cases}
$$

Note that the conjunction of an empty set of conjuncts is $t t$ while the disjunction of an empty set of disjuncts is $\neg t t$. It is easy to see that each temporal operator $\mathrm{X}$ in $\varphi_{s}$ is in front of $\mathrm{F}$ or $\mathrm{G}$. If we replace all occurrences of $\mathrm{XF}$ by $\mathrm{F}_{\mathrm{s}}$ and all occurrences of $X G$ by $G_{s}$, we always get formulae of $\operatorname{LTL}\left(F_{s}, G_{s}\right)$.

Finally, we define the formula $\varphi_{\mathcal{A}}$ equivalent to the whole automaton $\mathcal{A}$ as

$$
\varphi_{\mathcal{A}}=\bigvee_{c \in I} \bigwedge_{s \in c} \varphi_{s}
$$

Hence, we have shown that the following theorem holds.

Theorem 2. For each $M M A A \mathcal{A}$ with an alphabet of the form $2^{A P^{\prime}}$, we can construct an $\operatorname{LTL}\left(\mathrm{F}_{\mathrm{s}}, \mathrm{G}_{\mathrm{s}}\right)$ formula $\varphi_{\mathcal{A}}$ such that $L(\mathcal{A})=L\left(\varphi_{\mathcal{A}}\right)$.

\section{B Bounding Sets}

Let $\mathcal{A}=\left(S, \Sigma, \delta_{\mathcal{A}}, I, F\right)$ be an MMAA or a limMMAA. Here we show how to compute the set $\mathcal{Z} \subseteq 2^{S}$ of configurations for which the generalized Rabin pairs $\mathcal{G R}_{Z}$ of the corresponding TGDRA are constructed. If $\rho$ is an accepting run of $\mathcal{A}$, then $\operatorname{Inf}_{s}(\rho)$ is a subset of states reachable from must-states. Further, for each $w \in L(\mathcal{A})$ there is an accepting run $\rho$ over $w$ such that, for each $f \in F$, either $f \notin \operatorname{Inf}_{s}(\rho)$, or $\rho$ uses a single non-looping transition of $f$ (infinitely often) and the selfloop for $f$. We say that these runs are modest.

The function $z: S \rightarrow 2^{2^{S}}$ recursively computes, for a given state $s$, the sets of states that can potentially be reached from $s$ (not necessary as one configuration) 
infinitely often by a modest run visiting $s$ infinitely often. If $s$ is a may-state, a modest run uses only one of the non-looping transitions of $s$ and possibly also the selfloop of $s$. If $s$ is a must-state, a modest run can use an arbitrary combination of transitions of $s$. Formally, $z$ is defined as

$$
z(s)= \begin{cases}\{\{s\}\} \otimes \bigcup_{\substack{s, \alpha, c) \in \delta_{\mathcal{A}}, \mathbf{z}(c) \\ s \notin c}} \text { if } s \notin \operatorname{must}(S) \\ \{\{s\}\} \otimes \bigcup_{C \subseteq \operatorname{targets}(s)} \mathbf{z}\left(\bigcup_{c \in C} c\right) & \text { if } s \in \operatorname{must}(S),\end{cases}
$$

where $\otimes$ is an auxiliary operation defined for each $W_{1}, W_{2} \subseteq 2^{S}$ as

$$
W_{1} \otimes W_{2}=\underset{\substack{c_{1} \in W_{1} \\ c_{2} \in W_{2}}}{\bigcup}\left\{c_{1} \cup c_{2}\right\}
$$

targets $(s)$ contains configurations reachable from $s$ in one step minus the $s$ itself, i.e.

$$
\operatorname{targets}(s)=\left\{c \in 2^{S \backslash\{s\}} \mid(s, \alpha, c \cup\{s\}) \in \delta_{\mathcal{A}}, \alpha \in \Sigma\right\},
$$

and $\mathbf{z}$ is an auxiliary function defined for each configuration as

$$
\mathbf{z}(c)=\bigotimes_{s \in c} z(s) \quad \text { and } \quad \mathbf{z}(\emptyset)=\{\emptyset\} .
$$

The function $y: S \rightarrow 2^{2^{S}}$ computes, for a given state $s$, the sets of states that can potentially be reached from $s$ (not necessary as one configuration) infinitely often by a modest run visiting $s$ at least once. Note that if $s$ is a must-state $z(s)=y(s)$. Formally, $y$ is defined as

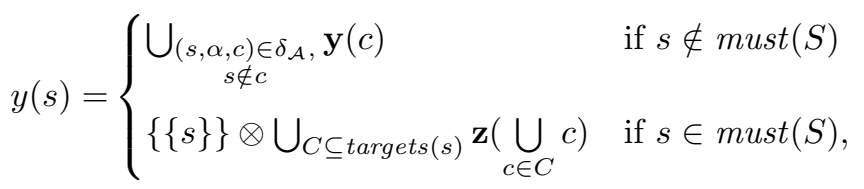

where $\mathbf{y}$ is an auxiliary function defined for each configuration as

$$
\mathbf{y}(c)=\bigotimes_{s \in c} y(s) \quad \text { and } \quad \mathbf{y}(\emptyset)=\{\emptyset\} .
$$

Finally, the set $\mathcal{Z}$ of configurations $Z$ for which we construct the generalized Rabin pairs $\mathcal{G R}_{Z}$ of the corresponding TGDRA is defined as $\mathcal{Z}=\bigcup_{c \in I} \mathbf{y}(c)$. Note that the states that are not successors of any must-state are not included in any element of the set $\mathcal{Z}$.

\section{More Experimental Results}

Table 3 provides experimental results for four parametric formulae, namely $\theta(n)=\neg\left(\left(\bigwedge_{i=1}^{n} \mathrm{GF} a_{i}\right) \rightarrow \mathrm{G}\left(b_{1} \rightarrow \mathrm{F} b_{2}\right)\right)$ of [14, its negation $\neg \theta(n), U(n)=$ $\left(\ldots\left(\left(a_{1} \cup a_{2}\right) \cup a_{3}\right) \ldots\right) \cup a_{n}$ of [15], and $U_{2}(n)=a_{1} \mathrm{U}\left(a_{2} \cup\left(\ldots \cup a_{n}\right) \ldots\right)$ of [15]. Note that $U(n)$ and $U_{2}(n)$ are outside the fragment LTL $(\mathrm{F}, \mathrm{G})$.

In all the cases but one $(U(5))$, LTL3DRA generates DRA of the same size as the other tools or smaller. Finally, LTL3DRA runs slower than lt12dstar on $U_{2}(n)$, while it is the fastest on the other three formulae. We list all instances of the formulae for which at least one of the tools produces a DRA before timeout. 


\begin{tabular}{|c|c|c|c|c|c|}
\hline \multirow{2}{*}{ Formula } & \multicolumn{2}{|c|}{ LTL3DRA } & \multicolumn{2}{|c|}{ Rabinizer } & 1tl2dstar \\
\hline & DRA $\mathrm{T}$ & TGDRA & DRA & GDRA & DRA \\
\hline$\theta(1)$ & $3(1)$ & 2 & $3(1)$ & 10 & $4(1)$ \\
\hline$\theta(2)$ & $4(1)$ & 2 & $5(1)$ & 20 & $8(1)$ \\
\hline$\theta(3)$ & $5(1)$ & 2 & $7(1)$ & 40 & $12(1)$ \\
\hline$\theta(4)$ & $6(1)$ & 2 & $9(1)$ & 80 & $16(1)$ \\
\hline$\theta(5)$ & $7(1)$ & 2 & $11(1)$ & 160 & $20(1)$ \\
\hline$\theta(6)$ & $8(1)$ & 2 & $13(1)$ & 320 & $24(1)$ \\
\hline$\theta(7)$ & $9(1)$ & 2 & timeor & & $28(1)$ \\
\hline$\theta(8)$ & $10(1)$ & 2 & timeol & & $32(1)$ \\
\hline$\theta(9)$ & 11(1) & 2 & timeol & & $36(1)$ \\
\hline$\theta(10)$ & $12(1)$ & 2 & timeol & & timeout \\
\hline$\theta(11)$ & $13(1)$ & 2 & timeor & & timeout \\
\hline$\theta(12)$ & 14(1) & 2 & timeor & & timeout \\
\hline$\neg \theta(1)$ & $6(3)$ & 2 & $8(3)$ & 10 & $7(2)$ \\
\hline$\neg \theta(2)$ & $12(4)$ & 2 & $16(4)$ & 20 & $13(3)$ \\
\hline$\neg \theta(3)$ & $24(5)$ & 2 & $32(5)$ & 40 & $25(4)$ \\
\hline$\neg \theta(4)$ & $48(6)$ & 2 & $64(6)$ & 80 & $49(5)$ \\
\hline$\neg \theta(5)$ & $96(7)$ & 2 & $128(7)$ & 160 & $97(6)$ \\
\hline$\neg \theta(6)$ & $192(8)$ & 2 & $256(8)$ & 320 & $193(7)$ \\
\hline$\neg \theta(7)$ & $384(9)$ & 2 & timeol & & $385(8)$ \\
\hline$\neg \theta(8)$ & $768(10)$ & 2 & timeor & & $769(9)$ \\
\hline$\neg \theta(9)$ & $1536(11)$ & 2 & timeol & & $1537(10)$ \\
\hline$\neg \theta(10)$ & $3072(12)$ & 2 & timeor & & $3073(11)$ \\
\hline$\neg \theta(11)$ & $6144(13)$ & 2 & timeor & & timeout \\
\hline$\overline{U(2)}$ & $3(1)$ & 3 & - & & $3(1)$ \\
\hline$U(3)$ & $5(1)$ & 5 & - & & $5(1)$ \\
\hline$U(4)$ & $9(1)$ & 9 & - & & $9(1)$ \\
\hline$U(5)$ & $24(1)$ & 24 & - & & $17(1)$ \\
\hline$U(6)$ & $68(1)$ & 68 & - & & timeout \\
\hline$U(7)$ & $212(1)$ & 212 & - & & timeout \\
\hline$U(8)$ & $719(1)$ & 719 & - & & timeout \\
\hline$\overline{U_{2}(2)}$ & $3(1)$ & 3 & - & & $3(1)$ \\
\hline$U_{2}(3)$ & $4(1)$ & 4 & - & & $4(1)$ \\
\hline$U_{2}(4)$ & $5(1)$ & 5 & - & & $5(1)$ \\
\hline$U_{2}(5)$ & $6(1)$ & 6 & - & & $6(1)$ \\
\hline$U_{2}(6)$ & $7(1)$ & 7 & - & & $7(1)$ \\
\hline$U_{2}(7)$ & $8(1)$ & 8 & - & & $8(1)$ \\
\hline$U_{2}(8)$ & $9(1)$ & 9 & - & & $9(1)$ \\
\hline$U_{2}(9)$ & $10(1)$ & 10 & - & & $10(1)$ \\
\hline$U_{2}(10)$ & 11(1) & 11 & - & & 11(1) \\
\hline$U_{2}(11)$ & $12(1)$ & 12 & - & & $12(1)$ \\
\hline$U_{2}(12)$ & $13(1)$ & 13 & - & & 13(1) \\
\hline$U_{2}(13)$ & timeout & & - & & $14(1)$ \\
\hline
\end{tabular}

Table 3. More parametric benchmarks. 